\title{
EFFECT OF WEIGHTED HAND M OVEM ENTS ON HAND FUNCTION IN CHRONIC STROKE PATIENTS
}

\author{
Hoda M. Zakaria ${ }^{1}$,Walaa M. Ragab ${ }^{2}$, Olfat I. Ali *3,
}

${ }^{1}$ Assistant professor at Department of physical therapy for neuromuscular disorders and its surgery, Faculty of Physical Therapy, Cairo University, Egypt.

${ }^{2}$ Lecturer at Department of physical therapy for neuromuscular disorders and its surgery, Faculty of Physical Therapy, Cairo University, Egypt.

*3 lecturer at Basic science department for Physical Therapy. Faculty of Physical Therapy, Cairo University. Egypt

\section{ABSTRACT}

Background: Hand dysfunction is a common problem of stroke patients and it is the main cause of impairment of the upper limb. Finding new method to improve hand performance will decrease the disability of chronic stroke patients.

Aim of the study: to study the effect of bilateral hand training with weight on the non paretic hand on the hand performance and time of performance in chronic stroke patients.

Materials and Methods: Thirty left chronic stroke patients participated in this study. The patients were assigned randomly into two equal groups. Group one (G1) received unilateral hand training and group two (G2) received bilateral hand training with weight on non affected hand. Both groups assessed two times before starting training program and after two months of training by Fugl meyer assessment scale, Wolf motor function test and hand dynamometer for the motor performance, time of performance and hand grip respectively.

Results: the patients in $\mathrm{G} 2$ showed significant improvement in the hand performance $(\mathrm{P}<0001)$ and significant decrease in the time of performance $(P<001)$ and also significant improvement of hand grip $(P<0001)$.

Conclusion: Bilateral hand movement with weight on the non affected hand has a significant effect on improving hand performance and decreasing the time of performance and increasing hand grip than unilateral hand movement.

KEY WORDS: bilateral hand training, hand function, stroke.

Address for correspondence: Dr. Olfat I. Ali, lecturer at Basic science department for Physical Therapy. Faculty of Physical Therapy, Cairo University. Egypt E-Mail: olfat_ib @ yahoo.com

\section{Access this Article online}

Quick Response code

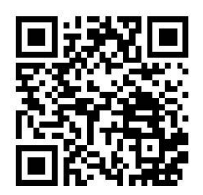

DOI: $10.16965 /$ ijpr.2016.214
International Journal of Physiotherapy and Research

ISSN 2321- 1822

www.ijmhr.org/ ijpr.html

Received: 20-12-2016 Accepted: 28-01-2017

Peer Review: 21-12-2016 Published (0): 11-02-2017

Revised: None

Published (P): 11-02-2017

\section{INTRODUCTION}

Voluntary movement control is typically impaired after a stroke. Wrist and finger extensors are a challenging aspect of upper-extremity recovery. Hand function is severely affected after stroke due to the high presentation of hand in cerebral cortex [1] More than half of the poststroke individuals continue with residual motor dysfunction as a long-term disability after the first year especially. The chronic motor problems lead to difficulty in the execution of functional movements especially hand functions [2].

Distal limb impairment is especially problematic, because proper hand function is crucial to 
manual exploration and manipulation of the environment. Loss of hand function is a major source of impairment in neuromuscular disorders and dependence in daily life [3].The severity of hand impairments and the negative impact on function are reasons for searching on new modalities or methods to rehabitate hand after stroke.

The two centrally linked arms form a coordinating structure when the upper extremities execute homologous coupling of muscle groups on both sides of the body [4]. Homologous movements with both arms and hands activate similar neural networks in both hemispheres $[5,6]$. Balanced interhemispheric interactions for normal voluntary movements support practicing bilateral arm movement and, consequently, facilitating the motor output from the damaged hemisphere. There are also strong temporal and spatial interactions between both arms such as frequency and phase locking as well as amplitude coupling [7]. Unilateral movements prevent mirror movements in the opposite upper limb. Forced-use unilateral movements may cause interhemispheric inhibition by increasing hyperexcitability of the unaffected motor cortex [8]. That's why we chose the bilateral hand movement for rehabilitation.

The changes of brain reorganization depend on the additional activity of the unimpaired limbs. Bilateral arm reaching with loading on the unaffected arm facilitates movement control on the affected upper limb in stroke with different levels of motor impairment. Loading the uninvolved limb serves as a useful adjunctive method of bilateral training in stroke and it still needs further experimental evidence. It was found that repeated measures model of walking with weight on the non affected leg led to significant improvement in walking speed, step length and cadence. Weight-bearing on the paretic leg increased also along with kinematics modifications including greater hip and knee excursion. These adaptations were maintained twenty minutes later after removing the mass. This study of weighting non affected leg supports the benefit of weighting non affected limb in improving kinematics and kinetics characters of the stoke limb [9]. So we added weight to non affected hand during bilateral hand movement.

\section{MATERIALS AND METHODS}

This study was conducted to assess the effect of bilateral weighted hand exercises on hand functions in patients suffering from stroke for more than six months. This study is a randomized control study.

Selection of the subjects: Thirty left chronic stroke patients represented the samples of the study. The patients were assigned randomly into two equal groups. All the patients were right handed and referred from a neurologist. The diagnosis was confirmed by MRI \&/or CT scan. The patients were selected from the Outpatient Clinic of Kaser El Aini, Teaching Hospital, Cairo University and from the Out-Patient Clinic, Faculty of Physical Therapy, Cairo University. All the patients were matched for age, duration of stroke and degree of hand motor impairment. The age of the chosen patients ranged from 50-65years old. All the patients had the ability to follow simple instructions and two step commands. The affected hand had a moderate motor impairment to Fugle - M yer scale [10]. After receiving an extensive explanation about the protocol, all the patients were given an informed written consent to the study.

The patients in group I (G1) were treated by unilateral hand training. All exercises were done from a sitting position. This type of exercise was chosen to increase the motor performance of the affected hand by concentration on increasing the activity of the affected hemisphere only. While the patients in group II (G2) were treated as in $\mathrm{G} 1$ but with bilateral weighted hand training. The weight was added on the non-paretic hand in form of weighted gloves. This type of exercise was chosen to increase the function of the affected hand by working on both hemispheres together to copy the process of motor performance of the affected hand from the non affected hemisphere to the affected one. The copying process was enhanced by increasing the excitability of the non affected hemisphere by adding weight. Also it was designed to balance the symmetry of both hemispheres.

The patients were excluded if they had symptomatic cardiac failure or unstable angina, uncontrolled hypertension ( $>190 / 110 \mathrm{~mm}$ $\mathrm{Hg}$ ), significant orthopedic or chronic pain 
conditions, active neoplastic disease, severe obstructive pulmonary disease, significant musculotendinous or bony restrictions of the affected upper limb like (Severe finger contractures that would preclude passive ROM of the hand), had an affected sided neglect, perceptuomotor or visual field deficits or apraxia and shoulder subluxation. A written informed consent approved by the Research Ethical Committee of the Faculty of Physical Therapy, Cairo University, was obtained from patients who participated in this study. And all procedures were conducted in accordance with the Declaration of Helsinki.

\section{Assessment tools of the subjects:}

All the patients were subjected to a complete neurological examination of detailed medical history, motor, sensory and ADL examination. Fugle-M eyer assessment scale (FM AS) was used to determine the severity of motor impairment. It is a valid and reliable test and also It correlates well with inter joint upper extremity (UE) coordination of stroke patients[11].

Wolf M otor Function Test (WM FT) was used also to time the movement. It is also a reliable test and is more sensitive than other UE tools. The timed items of (WM FT) assess the speed of performance so the changes in the temporal and spatial parameters of hand could be detected by this test. The non affected side was tested first followed by the affected side. Each patient was asked to apply each item of (WMFT) as quickly as possible and truncated at 120 seconds [11].

The hand-held dynamometer (HHD) (Penny and Giles, Biometrics Division, Blackwood Gwent NP2 IYD United Kingdom) measures the peak force produced by hand grip. This instrument is scored using force production: kilograms or pounds Kilograms (0-90), Pounds (0-200). It is a reliable and objective tools for measuring hand grip. These assessment tools were done two times for each patient; before beginning the treatment (pre test) and immediately after two months of the training; three times per weak (post test).

Procedure: All the patients in the two groups received the inhibitory techniques first for the spastic muscles. These techniques were applied in the form of a prolonged stretch to relax the spastic hand muscles and relaxation technique also. All the patients were treated from a sitting position with the hand relaxed on a front table. The course of training of each group was applied three times per week for six weeks (eighteen sessions). The researcher helped the patient to complete the designed task as needed. The speed and the level of the task maintained and not increased until the patients managed to perform it alone without any assistance.

Training procedures for $\mathrm{G1}$ based on wrist and hand movement of FMAS: First exercise was from 90 degree elbow flexion and zero degree shoulder position. To enhance the wrist stability the patients tried to extend the affected wrist to 15 degree and maintain this position if possible against resistance then the patient tried to extend and flex the affected wrist. Second exercise was from zero degree elbow and thirty degree shoulder position. To enhance the wrist stability the patients tried to extend the affected wrist to 15 degree and maintain this position against resistance then the patient tried to extend and flex the affected wrist. Third exercise was to perform circumduction movement of the wrist. Then hand exercises on form of flex and extend fingers, adduct and abduct thumb, thumb opposition to index, cylindrical and spherical grasp was performed by the affected hand only with assistance of physiotherapist to complete each one.

\section{Training procedures for $\mathbf{G} 2$ based on wrist and hand movement of FM AS: This group received} the same exercises for $\mathrm{Gl}$ but all exercises performed bilaterally with wearing weighted designed gloves on the non affected hand. The designed weighted gloves were made of wool but filled with sand to give resistance but not to cause fatigue.

Data analysis and statistical design [12]: The wilcoxon matched pairs test, it tests whether the samples originate from the same distribution. This test is similar to the parametric paired T test. It is used for comparing between two dependent samples. wilcoxon matched pairs test was used in this study to detect the significant difference within each group in the motor of performance and the time of motor performance 
from FM AS and WM FT. Mann-whitney U test, it tests whether the samples originate from the same distribution. This test is similar to the parametric unpaired T test. It is used for comparing between two independent samples. Mannwhitney $U$ test was used in this study to detect the significant difference between two groups in the motor of performance and the time of motor performance from FMAS and WMFT. Paired T test and unpaired T test was used to detect significant changes within groups and between groups respectively. SPSS for Windows Version 17 was used for all statistical analyses .All statistical procedures were two-tailed with significance set at á level less than 0.05 .

\section{RESULTS}

This study was conducted to determine the physical effects of two different types of physical therapy training on the hand functions in the chronic stroke patients. The best approach of physical therapy training for the hand functions could be determined from this study based on the impact of these approaches on the affected the hand functions by improvement of hand performance and timethrough assessment by FM AS and WMFT.

General demographic data of the patients of group I (G1) and group II (G2): The mean value of age in $\mathrm{G} 1$ and $G 2$ were $52.67 \pm 5.50$ and $52 \pm$ 6.4 years respectively. The mean value of stoke duration in $G 1$ and $G 2$ were $10.57 \pm 2.08$ and $10.29 \pm 2.98$ months respectively. Comparison of the mean values of the age and duration of stroke between Gland $G 2$ revealed no significant differences in the mean values of age or duration of stroke between the two groups ( $G 1$ and G2) ( $P \varangle 0.76$ for age and $P \varangle 0.77$ for duration of illness)

The effect of the two different training approaches within each group and between the two groups on Fugle-M eyer assessment scores:

\section{A- The mean values of motor performance of} pre and post tests in each group: The mean values of motor performance of $\mathrm{Gl}$ at pre and post tests were $10 \pm 1.6$ and $10.8 \pm 1.8$ respectively. The mean values of motor performance of $\mathrm{G} 2$ at pre and post tests were $9.4 \pm 0.55$ and $12.8 \pm 0.84$ respectively. Comparison of the mean values of motor performance of $\mathrm{G} 1$ and $\mathrm{G} 2$ at pre and post tests showed non significant changing of motor performance in $\mathrm{Gl}(\mathrm{P}<.51)$ and significant increase in the motor performance of the hand in G2 $(\mathrm{P}<.0001)$ (Table 1$)$.

B- Comparison of the mean values of motor performance of pre and post tests between the two groups: Comparison of the mean values of motor performance at $\mathrm{G} 1$ and $\mathrm{G} 2$ showed no significant difference of motor performance of hand between the two groups at pre test $(P<36)$ (Table 1). While, Comparison of the mean values of motor performance of hand at G1 and G2 showed significant difference of motor performance at post test between the two groups with more improvement of hand function for $G 2$ at post test $(P<0001)$ (Table 1$)$.

Table 1: The mean values of FM AS for hand section of each group.

\begin{tabular}{|c|c|c|c|c|}
\hline \multirow{2}{*}{$\begin{array}{c}\text { FM S for hand } \\
\text { section }\end{array}$} & \multicolumn{2}{|c|}{ Mean \pm SD } & \multirow{2}{\text{Mann-whitneyU}}{$\begin{array}{c}\text { P value } \\
\text { test }\end{array}$} & \\
\cline { 2 - 3 } & G1 & G2 & \\
\hline Pre test & $10 \pm 1.6$ & $9.4 \pm 0.55$ & 0.906 & 0.36 \\
\hline Post test & $10.8 \pm 1.8$ & $12.8 \pm 0.84$ & 7.368 & 0.0001 \\
\hline Wilcoxon test & 0.667 & 7.111 & & \\
\hline P value & 0.51 & 0.0001 & & \\
\hline
\end{tabular}

$\mathrm{SD}=$ Standard deviation $\mathrm{p}$ value: significance level, G1: group (1), G2: group (2)

It is to be concluded that the results of FMAS for the motor performance of the hand indicated that the training program of G2 only had a significant effect on increasing the motor performance of the affected hand in the chronic stroke patients.

The effect of the two different training approaches within each group and between the two groups on wolf motor function test for the time of motor performance and on hand dynamometer for the hand grip: The mean values of the time of motor performance of $\mathrm{Gl}$ at pre and post tests were $1547 \pm 42$ and $1544 \pm 42$ respectively. Comparison of the mean values of the time of motor performance of $\mathrm{G} 1$ at pre and post tests showed no significant changing for the time of motor performance over the period of assessment $(\mathrm{P}<06)$ (Table 2$)$.

The mean values of the time of motor performance of $\mathrm{G} 2$ at pre and post tests were $1555 \pm 41$ and $961 \pm 47$ respectively. Comparison of the mean values of the time of motor performance of $\mathrm{G} 2$ at pre and post tests showed a significant 
changing for the time of motor performance over the period of assessment $(\mathrm{P}<001)$ (Table 2 ).

Comparison of the mean values of time of motor performance of pre test at $\mathrm{G} 1$ and $\mathrm{G} 2$ showed no significant difference for the time of motor performance between the two groups $(P<85)$ (Table 2).Comparison of the mean values for the time of motor performance of post test at $G 1$ and $G 2$ showed a significant difference for the time of motor performance between the two groups with less time of performance for $\mathrm{G} 2(\mathrm{P}<001)$ (Table 2).

Table 2: The mean values of WM FT (time of performance) of each group.

\begin{tabular}{|c|c|c|c|c|}
\hline \multirow{2}{*}{$\begin{array}{l}\text { WMFT time of } \\
\text { performance }\end{array}$} & \multicolumn{2}{|c|}{ Mean $\pm S D$} & \multirow{2}{*}{$\begin{array}{c}\text { Mann-whitney U } \\
\text { test }\end{array}$} & \multirow{2}{*}{$P$ value } \\
\hline & G1 & G2 & & \\
\hline Pre test & $1547 \pm 42$ & $1555 \pm 41$ & 0.183 & 0.85 \\
\hline Post test & $1544 \pm 42$ & $961 \pm 47$ & 43.553 & 0.001 \\
\hline Wilcoxon test & 2 & 32.661 & & \\
\hline$P$ value & 0.06 & 0.001 & & \\
\hline
\end{tabular}

$\mathrm{SD}=$ Standard deviation $\mathrm{p}$ value: significance level, $\mathrm{G} 1$ : group (1), G2: group (2)

Table 3: The mean values of hand grip of each group.

\begin{tabular}{|c|c|c|c|}
\hline \multirow{2}{*}{ Hand grip } & \multicolumn{2}{|c|}{ Mean \pm SD } & \multirow{2}{*}{ P value } \\
\cline { 2 - 3 } & G1 & G2 & \\
\hline Pre test & $3.63 \pm 1.27$ & $3.6 \pm 1.1$ & 0.95 \\
\hline Post test & $3.98 \pm 1.4$ & $5.8 \pm 1.12$ & 0.001 \\
\hline P value & 0.48 & 0.0001 & \\
\hline
\end{tabular}

$\mathrm{SD}=$ Standard deviation $\mathrm{p}$ value: significance level, $\mathrm{G} 1$ : group (1), G2: group (2)

It is to be concluded that the results of WMFT for the time of motor performance indicated that training program of $\mathrm{G} 2$ had a significant effects on decreasing the time of motor performance of the affected hand in the chronic stroke

The mean values of hand grip of pre and post tests in each group and between groups: The mean values of hand grip of $\mathrm{Gl}$ at pre and post tests were $3.63 \pm 1.27$ and $3.98 \pm 1.4$ respectively. The mean values of hand grip of $\mathrm{G} 2$ at pre and post tests were $3.6 \pm 1.1$ and $5.8 \pm 1.12$ respectively. Comparison of the mean values of hand grip of $G 1$ and $G 2$ at pre and post tests showed non significant changing of hand grip in $\mathrm{Gl}$ $(P<.265)$ and significant increase in the hand grip of the hand in G2 $(\mathrm{P}<.029)$ (Table 3$)$.

Comparison of the mean values of hand grip between $G 1$ and $G 2$ showed no significant difference of hand grip of hand between the two groups at pre test $(\mathrm{P}<341)$ (Table 3$)$. On the other hand,Comparison of the mean values of hand grip between $\mathrm{G} 1$ and $\mathrm{G} 2$ at post test showed significant increase of hand grip for $G 2$ $(\mathrm{P}<025)$ (Table 3).

\section{DISCUSSION}

The present study was conducted to assess the effect of the two different training programs on the motor performance of the affected hand in the chronic stroke patients. Forty five right handed patients suffering from left chronic stroke with hand dysfunction participated in this study. All the patients were matched for age, duration of stroke and degree of motor impairment. The patients were assigned randomly into two equal groups; the group I (G1) and the group II (G2). The patients in G1 received unilateral arm training while the patients in $\mathrm{G} 2$ received bilateral weighted arm training with weight on the non affected hand.

The selection of the patients was depended on the side of the stroke. All the patients were left hemiplegia so all the patients in the current study were matched for the site of stroke. The function of the left brain differs from the right one and this affects the level of the motor performance of UE and hand in the chronic stroke patients. The side of lesion has also an impact on the degree of plasticity. It was found that the left hemisphere is concerned with analytical processing of individual components, sequencing of tasks, and language. The right hemisphere is more focused on the perception of whole and spatial tasks. Person with left hemisphere were also able to make less use of sensory information to update and modify the direction of the movement than persons with right hemisphere damage because the left hemisphere is responsible for motor programming[13-15].

In the current study all the selected patients were right handed dominance. It was proved that the handiness of the stroke patients affects the individual's functional ability and the rate of neural plasticity. Left-handed dominant people perform some tasks with the right hand. The right-handed dominant individuals rarely perform tasks primarily with the left hand and the rate of recovery decreases because of increasing the learning of the non use[16,17]. 
So all the patients in the current study were right handed.

The results of the present study proved a significant effect of bilateral arm training with weight on the affected hand performance. This might attributed to bilateral arm training is a good method of increasing the neural connection and integration which is a part of the neural plasticity and motor learning. This is agreed by $[18,19]$.

Ameli et al.(2009) explained the physiological effect of bilateral arm training which might explain why patients in group two of our study showed significant improvement in hand performance and decreasing in the time of motor performance. [20]. Cerebral blood flow of the ipsilesional motor cortex is increased after decreasing the excitability of the unaffected hemisphere which occurs by bilateral arm training and that is increased if weight is added to non affected limb. This results in changing in the metabolic function of the brain and promoting the neural plasticity and motor recovery after stroke. This is agreed also by [21-24].

Bilateral symmetrical tasks with weight augment the performance of the paretic limb and the kinematic variables as the time of performance. This is agreed by [25-27]. When the impaired and non-impaired hands are moved symmetrically, crossed facilitatory drive from the intact hemisphere will produce increased excitability in homologous motor pathways in the impaired hand. The improvement in the current study may be due to the Kinematic features of each hand motion that are found in the trajectory of the other hand. The trajectories become spatially $\& /$ or temporally coupled. Neural processes that mediate interlimb interactions can be accounted for by informational exchanges at the different levels of the CNS, resulting in neural crosstalk and finally, in kinematic coupling of limb or hand trajectories. The nonparetic hand would exhibit temporal adaptation to that of the paretic hand in both anticipatory and motor control domains for a bimanual symmetrical weighting task. This is consistent with [28].

The relationship between the motor neuron size and the number and size of the muscle fibers it innervates is usually lost or damaged after Int J Physiother Res 2017;5(1):1885-92. ISSN 2321-1822 stroke. Reorganization of this relationship at motor endplates occurs over time through axon branching and neuromuscular activity .This reorganization, known as neuroplasticity, occurs as the nervous system attempts to repair the damaged motor neurons. Bilateral arm training with weight increases the reorganization of this relationship at motor endplates occurs over time through the axonal branching and neuromuscular activity so the time of performance of the hand decreases as in the current study. This is agreed by [29-31].

The result of the present study contradicts with $[32,33]$ The authors revealed that unilateral arm training produced greater functional gains and use of the paretic arm in daily life than bilateral arm training. The unilateral arm training group also obtained a higher improvement in WM FTTime score than the bilateral arm training group, thus demonstrating larger improvements in the temporal efficiency of motor function. The contradiction of these results with the results of the current study may be due working on small sample sizes and using bilateral arm training of asymmetrical type not symmetrical type with weight as in the current study. The difference in the patients' age may be also contributed for this contradiction.

\section{CONCLUSION}

Bilateral arm training of the hand with weight on the non affected hand has a good effect on improving the hand function and decreasing the time of performance of the affected hand in chronic stroke patients.

\section{ACKNOW LEDGEM ENTS}

Authors would like to thank all patients participate in this study.

\section{Conflicts of interest: None}

\section{REFERENCES}

[1]. Cauraugh James, Kathye Light, Sangbum Kim, Mary Thigpen , Andrea Behrman. Chronic Motor Dysfunction After Stroke Recovering Wrist and Finger Extension by Electromyography-Triggered Neuromuscular Stimulation. Stroke. 2000;31:1360-1364.

[2]. Heidi C. Fischer, Kathy Stubblefield, Tiffany Kline, Xun Luo, Robert V. Kenyon, Derek G. Kamper. Hand Rehabilitation Following Stroke: A Pilot Study of Assisted Finger Extension Training in a Virtual Environment. Top Stroke Rehabil. 2007;14(1):1-12. 
[3]. Deutsch JE, Merians AS, Adamovich S, Poizner H, Burdea GC. Development and application of virtual reality technology to improve hand use and gait of individuals post-stroke. Restor Neurol Neurosci. 2004;22(3-5):371-386.

[4]. Kyoung Ju Han and Jin Young Kim. The effects of bilateral movement training on upper limb function in chronic stroke patients. J Phys Ther Sci. 2016;28(8): 2299-2302.

[5]. Mudie MH , M atyas TA. Can simultaneous bilateral movement involve the undamaged hemisphere in reconstruction of neural networks damaged by stroke? Disabil Rehabil. 2000;22:23-37.

[6]. Lin Jau-Hong, Hsu Miao-Ju, Sheu Ching-Fan, Wu TzungShian, Lin Ruey-Tay, Chen Chia-Hsin , Hsieh ChingLin. Psychometric Comparisons of 4 M easures for Assessing Upper-Extremity Function in People With Stroke. PHYSTHER. 2009;89:840-850.

[7]. Cauraugh James H, Coombes Stephen A, Lodha Neha, Naik Sagar K, Summers Jeffery J. Upper extremity improvements in chronic stroke: Coupled bilateral load training. Restor Neurol Neurosci. 2009;27(1) :17-25.

[8]. Debaere F, Wenderoth $N$, Sunaert S, Van Hecke P, Swinnen SP. Changes in brain activation during the acquisition of a new bimanual coordination task. Neuro psychologia .2004;42:855-867.

[9]. RegnauxJP, Pradon D, Roche N, Robertson J , Bussel $B$, Dobkin B. Effects of loading the unaffected limb for one session of locomotor training on laboratory measures of gait in stroke. Clinical Biomechanics.2008;23(6):762-768.

[10]. Velozo Craig A, Woodbur Michelle L. Translating measurement findings into rehabilitation practice: An example using Fugl-M eyer Assessment-Upper Extremity with patients following stroke. Journal of rehabilitation research and development(JRRD). 2011;48(10):1211-1222.

[11]. Cauraugh James H, Lodha Neha, Naik Sagar K, Summers Jeffery J. Bilateral M ovement Training and Stroke M otor Recovery Progress: A Structured Review and M eta-Analysis. Hum M ovSci .2010;29(5): 853-870.

[12]. Marusteri Marius, Bacarea Vladimir. Comparing groups for statistical differences: how to choose the right statistical test? Biochemia Medica 2010;20(1):15-32.

[13]. Wu CY, Trombly CA, Lin KC, Tickle Degnen L. A kinematic study of contextual effects on reaching performance in person with and without stroke: influences of object availability. Arch Phys M ed Rehabil. 2000;81:95-101.

[14]. Levy CE, Nichols DS, Schmalbrock PM, Keller P, Chakeres DW. Functional M RI evidence of cortical reorganization in upper-limb stroke hemiplegia treated with constraint-induced movement therapy. Am J Phys Med Rehabil. 2001;80:4-12.

[15]. Ströckens F, Güntürkün O, Ocklenburg S. Limb preferences in non-human vertebrates. Laterality .2013;18:536- 575 .
[16]. Langan J , Van Donkelaar P. The influence of hand dominance on the response to a constraint-induced therapy program following stroke. Neurorehabil Neural Repair. 2008;22(3):298-304.

[17]. Takeuchi Naoyuki , Oouchida Yutaka , Izumi ShinIchi . Motor Control and Neural Plasticity through Interhemispheric Interactions. Neural Plasticity. 2012;10:1155-13.

[18]. Van Der Knaap LJ, Van Der Ham IJM. How does the corpus callosum mediate interhemispheric transfer? A review. Behavioural Brain Research.2011; (223)1:211-221.

[19]. Nowicka A, Tacikowski P. Transcallosal transfer of information and functional asymmetry of the human brain. Laterality.2011;16(1):35-74.

[20]. Ameli M , Grefkes C, Kemper F, Riegg FP, Rehme AK, Karbe $H$, Fink GR, Nowak DA. Differential effects of high-frequency repetitive transcranial magnetic stimulation over ipsilesional primary motor cortex in cortical and subcortical middle cerebral artery stroke. Annals of Neurology.2009;66(3):298-309.

[21]. Takeuchi N, Tada T, Toshima M, Chuma T, M atsuo Y, Ikoma K. Inhibition of the unaffected motor cortex by $1 \mathrm{HZ}$ repetitive transcranial magnetic stimulation enhances motor performance and training effect of the paretic hand in patients with chronic stroke. Journal of Rehabilitation Medicine. 2008;40(4):298-303.

[22]. Rizzo V, Siebner HR, Morgante F, Mastroeni C, Girlanda P, Quartarone A. Paired associative stimulation of left and right human motor cortex shapes interhemispheric motor inhibition based on a hebbian mechanism. Cerebral Cortex. 2009;19(4): 907-915.

[23]. Takeuchi N, Ikoma K. 1 Hz rTM S over unaffected hemisphere in stroke patients alters bilateral movements and coupling between motor areas. Clinical Neurophysiology. 2010;121(1):316.

[24]. Di Lazzaro V, Profice P, Pilato F, Capone F, Ranieri F, Pasqualetti P, Colosimo C, Pravatà E, Cianfoni A ,Dileone M. M otor cortex plasticity predicts recovery in acute stroke. Cerebral Cortex.2010;20(7): 1523-1528.

[25]. M cCombe Waller S, Harris-Love M, Liu W , Whitall J. Temporal coordination of the arms during bilateral simultaneous and sequential movements in patients with chronic hemiparesis. Exp Brain Res. 2006;168:450-454.

[26]. Stoykov M E, Lewis GN , Corcos DM . Comparison of bilateral and unilateral training for upper extremity hemiparesis in stroke. Neurorehabil Neural Repair .2009;23:945-953.

[27]. Lin Chorng-Horng, Chen Michael, Sun Mu-Chien. Circulating apoptotic factors in patients with acute cerebral infarction.clinical biochemistry.2010; 43(9):761-763.

[28]. Kwakkel G, Kollen BJ, Krebs HI. Effects of robot-assisted therapy on upper limb recovery after stroke: a systematic review. Neurorehabil Neural Repair. 2008;22:111-121. 
[29]. Cauraugh JH, Kim SB. Two coupled motor recovery protocols are better than one: electromyogram-triggered neuromuscular stimulation and bilateral movements. Stroke. 2002;33:1589-1594.

[30]. Van Dieen JH, Futoshi O, De Haan A. Reduced neural drive in bilateral exertions: A performance-limiting factor. Med Sci Sports Exerc. 2003;35(1):111-118.

[31]. Yang Chieh-Ling, Lin Keh-Chung, Chen Hsieh-Ching, Wu Ching-Yi, Chen Chia-Ling. Pilot Comparative Study of Unilateral and Bilateral Robot-Assisted Training on Upper-Extremity Performance in Patients With Stroke. American Journal of Occupational Therapy.2012;66:198-206.
[32]. M cCombe Waller S, Liu W , Whitall J. Temporal and spatial control following bilateral versus unilateral training. Hum M ov Sci. 2008;27:749-758.

[33]. Wu CY, Chuang LL, Lin KC, Chen HC , Tsay PK. Randomized trial of distributed constraint-induced therapy versus bilateral arm training for the rehabilitation of upper-limb motor control and function after stroke. Neurorehabil Neural Repair. 2011;25:130-139.

\section{How to cite this article:}

Hoda M. Zakaria, Walaa M. Ragab, Olfat I. Ali. EFFECT OF WEIGHTED HAND M OVEM ENTS ON HAND FUNCTION IN CHRONIC STROKE PATIENTS. Int J Physiother Res 2017;5(1):1885-1892. DOl: 10.16965/ijpr.2016.214 